\title{
Sistemas locales de justicia como respuesta a la desarticulación institucional en las entidades territoriales del Estado colombiano
}

\author{
Local justice systems as a response to institutional disarticulation in the \\ territorial entities of the Colombian State \\ Humberto de Jesús Herrera Ortega ${ }^{1}$ \\ Margarita Rodelo Garcia²
}

\begin{abstract}
RESUMEN
A lo largo de este artículo, se estructuran tópicos relacionados con la implementación y regulación de los Sistemas Locales de Justicia, siendo este una estrategia colectiva de resolución de conflictos y garantía de acceso a la justicia, orientado en la recuperación de confianza por parte de las comunidades hacia las autoridades que administran justicia Para el estudio de este marco jurídico se tuvieron en cuenta el desarrollo de mecanismos análogos como antecedentes de implementación, así como la experiencia en territorio de la aplicabilidad de dicho sistema
\end{abstract}

\section{Palabras clave:}

Sistemas Locales de Justicia, Conflicto Armado, Cooperación internacional, Marco Jurídico.

\footnotetext{
${ }^{1}$ Abogado de la Universidad Libre, Especialista en Derecho Constitucional de la Universidad libre, Asesor de la Contraloría Distrital de Barranquilla y Director Administrativo de la Corporación CEPAD, h.he_10@hotmail.com humbertod-herrerao@unilibre.edu.co

${ }^{2}$ Abogada de la Universidad Rafael Núñez, Master en Derecho Constitucional de la Universidad de Valencia España, Especialista en Derecho Administrativo de la Universidad Libre seccional Barranquilla. Docente Universidad Dirección Nacional de Escuelas, Asesora de la Contraloría Distrital de Barranquilla y directora de la Fundación Proyecto Alas, rosa.rodelo@correo.policia.gov.co; rodelo2@alumni.uv.es.
}

Recibido: 25 de septiembre de 2019. Aprobado: 15 de noviembre de 2019. 


\begin{abstract}
In the development of this article, topics related to the implementation and regulation of the local systems of justice are structured, being this a collective strategy of conflict resolution and guarantee of access to the justice, oriented in the Recovery of confidence by the communities towards the authorities that administer justicet. For the study of this legal framework, we took into account the development of similar mechanisms as a history of implementation and the experience in territory of the applicability of that system.
\end{abstract}

\title{
Key words:
}

Local Justice Systems, Conflict Armed, International Cooperation, Legal Framework

\section{Introducción.}

En el marco del posconflicto y del acuerdo de paz, Colombia se encuentra inmersa en una etapa de transición que tiene su fundamento en la necesidad de adecuar un modelo Jurídico a los nuevos retos que implica la terminación de un conflicto. Parte de este desafío es diseñar herramientas e iniciativas que construyan paz en los territorios, entendiendo paz territorial como aquello que debería pasar normalmente en zonas vulnerables.

Los sistemas locales de justicia, cuyo estudio se debate en el presente artículo científico, son precisamente una manifestación de las estrategias o herramientas tendientes a garantizar y efectivizar lo que hasta ahora sólo ha sido una utopía, esto es, la ya mencionada paz territorial.

Si bien, la estrategia de implementación de los Sistemas Locales de Justicia es un proceso de construcción de más de 10 años, es este momento histórico el que 
permite atender las conflictividades de los Municipios sin presencia de grupos armados, lo que da vía libre a un resultado eficaz. Para ello es importante la compresión de sistemas paralelos que ayudan a darle una vía distinta al trámite ordinario y que lleven consigo una recuperación en la confianza estatal, pues no solo se trata de tener los mejores métodos, sino que las personas recurren al Estado como medio de respuesta a sus conflictos.

Básicamente este sistema permite que las autoridades locales trabajen de manera articulada y conjunta en las necesidades jurídicas de un Municipio. Esto implica no solo el desarrollo de una justicia con autoridades a nivel Judicial y administrativo, también involucra la intervención de la justicia comunitaria, es decir, el rol educativo de las instituciones académicas y la formación de conciliadores en equidad como medio formal de respuesta a los interrogantes y problemas de una comunidad (Ardila, É. 2015, P. 5).

A través del programa manos a la paz, iniciativa de la alta consejería para el posconflicto y PNUD (Programa de Naciones Unidas para el Desarrollo), se pudieron adquirir de manera directa conocimientos en la aplicación del para el desarrollo de este artículo, entiendo que el programa otorgó la posibilidad de ir a una zona de conflicto, en este caso Lejanías - Meta, para trabajar en la implementación de los Sistemas Locales de Justicia, lo que permitió la obtención de una caja de herramientas para la configuración y el estudio de cada elemento importante en la ejecución del plan de trabajo.

Dicha caja de Herramientas permite delimitar el alcance de la competencia o capacidad de los actores que tienen la potencialidad de participar en el Sistema y de cómo deben ser implementados los SLJ en los Municipios (Ardila, E. 2015, P. 5). 
En ese orden de ideas, el presente trabajo tiene como propósito profundizar a través de la experiencia adquirida, el funcionamiento del sistema como medio alternativo y funcional de acceso a la justicia, situación que permite atender de manera legítima los conflictos enmarcados dentro del aparato judicial, además del estudio de sus antecedentes normativos y prácticos, así como la modalidad bajo la cual funcionaban, mostrando los cambios estructurales ocasionados por estos dentro de los municipios que hoy son ejemplo en materia de aplicación.

El método deductivo bajo el entendido de que se ha elaborado un análisis general en primera medida, sobre los antecedentes y el marco normativo de la administración de justicia de manera alternativa en Colombia y las distintas estrategias de respuesta rápida en la solución de conflictos, para luego remitir el desarrollo al marco particular en la implementación de los Sistemas Locales de Justicia.

\section{Antecedentes.}

Para orientar un análisis al entendimiento de la iniciativa en la implementación de métodos alternativos en función de mejorar la administración de justicia, y en este caso, de los Sistemas Locales de Justicia, su creación tiene su razón de ser en la poca creencia estatal como medio resolutivo de conflictos además de la emergente justicia paralela en la etapa de conflicto armado en Colombia, de ahí que desde hace más de una década se trabaje en la diversificación de la oferta de instituciones que busquen promover medios formales o no formales en la eficacia resolutiva de las controversias. 
Si bien en Colombia siempre ha existido una oferta institucional, la poca capacidad de ejecución en el territorio nacional le ha quitado peso y campo de acción en muchas zonas donde el estado representa una figura ausente y sin ningún peso en las conflictividades de la zona urbana y rural. Esa problemática de desarticulación y carencia sistemática de los actores locales da resultados alarmantes en materia de justicia, lo que genera índices y tasas altas de poca efectividad en el desarrollo normativo y capacidad de respuesta rápida.

Existen varias situaciones que dan cuenta del problema de acceso a la justicia en Colombia, pero generalmente éste está representado en el alto grado de congestión de procesos que sufren los Juzgados y Tribunales, hecho que da lugar a la ineficacia y a la dilatación en la resolución de los conflictos jurídicos, sobre este punto, la Corporación excelencia en la Justicia manifestó lo siguiente:

"Los jueces comenzaron este año con 1'231.000 procesos rezagados en lo penal, laboral, lo civil y de familia, Esto significa, según la Corporación, que en promedio un juez se atrasa un 10 por ciento con los casos que recibe en un año. El indicador internacional está por el 5 por ciento, como máximo". (Corporación Excelencia en la Justicia, 2017)

Lo anterior ocasiona des legitimidad y le resta credibilidad a las instituciones estatales, creando en el ciudadano común, un imaginario según el cual, las entidades públicas sólo son una fachada burocrática incapaz de contribuir a la construcción de paz territorial a través de la coordinación y la cooperación en la solución de controversias que son inherentes a toda sociedad.

Para contrarrestar esta situación indeseable se han creado planes de acción de origen normativo, que persiguen la plenitud de la eficacia en la administración de justicia, una muestra de eso es el Plan especial de descongestión de la jurisdicción 
de lo Contencioso Administrativo, emanado de la sala administrativa del Consejo Superior de la Judicatura. (Consejo Superior de la Judicatura, 2012).

Por otra parte, es menester mencionar los efectos negativos que en materia de confianza institucional ha tenido el conflicto armado interno, quienes además de lesionar los bienes jurídicos constitucional y convencionalmente protegidos, a través de coacción y el empleo de la fuerza han usurpado y reemplazado el poder soberano del Estado en la facultad de administrar justicia, fenómeno que es denominado como, Justicia Paralela. Esta anomalía o situación atípica tiene como principal causa la ausencia del Estado y la debilidad institucional en los territorios periféricos alejados del centro del país, toda vez que, los vacíos o espacios de las autoridades legítimas son ocupados por otros actores que generalmente son grupos armados al margen de la ley.

Queda ilustrado el concepto de justicia paralela, donde son estos grupos los que administran justicia, incluso es la propia ciudadanía, la que aboga, avala y justifica tal usurpación de facultades, como quiera que, para ellos resultaba más eficaz esta resolución porque sus problemas eran resueltos con inmediatez, situación de la cual fue testigo el autor del presente trabajo de investigación siendo participe en la implementación de los sistemas en Lejanías- Meta.

Como justificación a ese factor problema, se intensificaron a lo largo de los años varias directrices a unificar el aspecto jurídico y operacional de las instituciones, que pudiesen dar garantía y resultado a esa situación, para ejemplificar el escenario anteriormente planteado, se pone de presente la creación de las casas de justicia, que pretenden aproximar la oferta institucional a la ciudadanía en el territorio. 
En ese orden de ideas, respecto del origen de la categoría, casa de justicia, el grupo de investigación de justicia plantea:

"El concepto de casa de justicia fue propuesto por el profesor de Harvard, Frank Sander en 1976, como un proyecto para articular las instituciones públicas dedicadas a prestar servicios de justicia estatal con los diversos mecanismos alternativos de solución de conflictos. En Colombia, la implementación de las casas de justicia inició a partir de mediados de la década de los noventa, y su reglamentación se realizó en el año 2000. Según el Decreto 1477 de 2000, las casas de justicia son centros en los que se reúnen distintas agencias estatales, nacionales y locales, para ofrecer de manera articulada servicios de justicia formal y no formal". García Villega. M, Espinosa. J, Lalinde. S, Arroyave. L, Villadiego. C. (2015).

Estas casas constituyeron una idea para el mejoramiento del acceso a la justicia, las destinadas a las personas más vulnerables económicamente. Bajo el marco anteriormente estructurado, explica la necesidad estatal de crear un modelo que responda a necesidades jurídicas y al problema de la desarticulación esquemática en las entidades territoriales.

A partir de ese postulado, el concepto organizacional de los Sistemas Locales de Justicia ha evolucionado con los años, pasando por los extintos Centros de Convivencia operados para llevar la labor articulada de todas las formas de resolución de conflictos en las zonas marginadas de los Municipios Pequeños y Medianos, hasta consolidarse en un proyecto de coordinación que garantiza la presencia de los operadores de justicia en el territorio municipal. 


\section{Los Sistemas Locales de Justicia}

Antes de esquematizar un concepto, es bueno esclarecer la percepción sobre la administración de justicia, en el sentido de quienes la ejercen. $Y$ es que la guía judicial no solo se deriva del campo de acción de los tribunales, juzgados o distintos entes a cargo de gestionar la demanda jurídica, sino también entes alternativos que cooperan en dicha administración, y en este aspecto se involucran figuras como el rol de la justicia comunitaria con los conciliadores en equidad, así como también el papel de la justicia educativa y su misión direccional de las nuevas generaciones en involucrarse en temas formativos y de carácter social. (Ardila, Edgar. 2017, P. 9)

En ese sentido, y basado en el documento elaborado por el Programa de Naciones Unidas para el Desarrollo (PNUD), en el marco del proyecto;

"Los Sistemas Locales Justicia, surgen como una Estrategia de Respuesta Rápida diseñada por el Gobierno Colombiano, a través de la Alta Consejería para el Postconflicto, los Derechos Humanos y la Seguridad, como un conjunto de iniciativas que fortalezcan la confianza en el Estado en el contexto de postconflicto. (Programa de Naciones Unidas para el Desarrollo PNUD, 2016

De igual forma el PNUD con este proyecto busca fomentar estrategias dando ese enfoque más territorial y de justicia, así lo indica en el documento del proyecto:

"La estrategia involucra diferentes proyectos, con enfoque y despliegue territorial en materia de justicia, seguridad, desarrollo, gobernabilidad y justicia transicional. (...) El objetivo principal del proyecto es mejorar el acceso a la justicia en municipios priorizados a partir de la creación y/o fortalecimiento de los Sistemas Locales de Justicia en trece (13) municipios priorizados en el marco de la Estrategia de Respuesta Rápida, en cuatro (4) departamentos del país: Antioquia, Guaviare, Meta y Norte de Santander. Los beneficiarios directos son los 13 Sistemas Locales de Justicia creados y fortalecidos en territorio; Revista Legem, Universidad del Atlántico 5 (2), Julio - Diciembre 2019, pp. 23-41 
autoridades, líderes comunitarios y operadores de los Sistemas Locales de Justicia fortalecidos en sus capacidades en 13 municipios priorizados de los departamentos de Antioquia, Guaviare, Meta y Norte de Santander; Inspectores de Policía de 30 municipios de Antioquia y 29 municipios del Meta fortalecidos en sus capacidades". (Programa de Naciones Unidas para el Desarrollo - PNUD, 2016).

Los Sistemas Locales de Justicia resultan una estrategia conjunta de trabajo, organizada y sistemática que responde a necesidades de justicia identificadas en las entidades territoriales, como quiera que su funcionamiento proporcione una garantía en la gestión del conflicto y gobernabilidad institucional (Buelvas y Flores, 2017)

Bajo el entendido que cada concepto de esta estrategia refleja un funcionamiento, resulta pertinente desglosar a qué obedece la noción de sistema local. Como primera medida, están representados en un sistema, porque se estructuran de manera organizada, esquemática y necesitan de la proactividad de cada funcionario local que de una u otra manera coopera en la respuesta de conflictos. La actuación como sistema por parte de los actores de justicia debe tener un alto grado de incidencia y que con su actuación contrarreste los efectos de la desarticulación. Ahora bien, el término local se emplea con el propósito de resolver el problema del centralismo en la realidad jurídica y estatal, permitiendo llevar a zonas de alto impacto en cuanto a conflictividades, la inclusión que implica esta estrategia con el fin de solventar los niveles de debilidad institucional. (Ardila, É. 2015, P. 20).

La implementación de los llamados sistemas locales de justicia proviene de un estudio previo sobre las condiciones sociales e institucionales de cada Municipio, lo que lleva a comprender sus particularidades y la manera es cómo pueden 
involucrarse las estrategia funciona de manera sistematizada, conlleva a entender que está constituida por unos elementos y que para que opere de manera exitosa, dichos elementos tienen que trabajar de manera articulada, y es donde los actores locales que eventualmente propiciarán ese liderazgo, toman un papel importante. No hay lugar a discutir los efectos positivos de este engranaje, si no hay apropiación de las problemáticas y no hay un impacto positivo en la transformación social de cada lugar.

\section{Integrantes y organización de los sistemas locales de justicia}

El sistema estará integrado, por varias figuras jurídicas con distintos roles en la administración de justicia. Si bien la funcionalidad, es que cada operador de esta estrategia desde el uso de sus funciones pueda de manera conjunta dirimir conflictos, cada funcionario genera un impacto distinto de acuerdo al grado de relevancia en sus decisiones, es por eso que tanto los jueces, las autoridades administrativas con funciones de administración de justicia son muy importantes, debido a que desde esa representación se deriva un buen liderazgo, así que el punto inicial es involucrar a estos actores como parte activa y consistente en la elaboración de las rutas y diagnóstico de cada municipio donde esté priorizado con el proyecto. En ese sentido la caja de herramientas elaborada por PNUD y profesor Ardila (2015), detalla el siguiente esquema organizacional:

Por un lado, encontramos que los operadores que deciden, y que tienen un rol más influyente en la estructuración del sistema en este rango están: Los Jueces, Autoridades indígenas, Árbitros, Autoridades administrativas con funciones de administración de Justicia, Inspectores de policía, comisarios de familia, conciliadores, corregidores y alcaldes" (p. 22). 
En un segundo según Ardila (2015) el orden por su parte se encuentran los Operadores Consensuales, que lo conforman; Fiscales, Defensores públicos, Defensores de menores, Medicina legal, Personeros, Policía, Ejército, Procuraduría, ICBF, Hospitales, Instituciones Educativas, Conciliadores en Derecho y en Equidad (p.22). Por otra parte, están las Redes Sociales y entidades promotoras, como las Partes en el conflicto, Comunidad, Instituciones, Redes.

$Y$ finalmente nos deja que las Entidades que impactan sobre la cultura en este punto se encuentran las Instituciones Educativas, Organizaciones de jóvenes o de mujeres, Comunidades de fe, Juntas de Acción Comunal, Sindicatos, Medios de comunicación, Asociaciones, Conciliadores. (Ardila, É. 2015, P. 22).

Bajo la premisa de que en las rutas de gestión de conflicto pueden concurrir, y asociarse varios operadores, además de las formas individuales en las actividades institucionales, la forma básica de organización del sistema será la que establecerá las rutas y protocolos de atención y de intervención en las conflictividades con los actores y los operadores implicados.

\section{Pluralismo jurídico como principio del sistema local de justicia}

Los sistemas locales de Justicia están guiados y orientados por unos principios que están llamados a marcar la ruta en la implementación de esta estrategia. En cuanto a la concepción de estos principios o normas fundamentales el marco conceptual del ministerio de Justicia ha dicho que:

"El Enfoque Sistémico pone en el centro las relaciones de confianza y de comunicación de todos los actores que hacen parte del sistema y el enfoque territorial busca fortalecer las capacidades locales en los 
territorios para mejorar el acceso a la justicia. A su vez, el desarrollo participativo, en este sentido, busca articular y fortalecer colectivos y organizaciones sociales existentes en las comunidades y el marco diferencial que las particularidades de cada territorio pasan por reconocer la diversidad, tanto en el enorme potencial de riqueza natural, social y cultural". (Ministerio de Justicia, 2017).

En ese orden de ideas, los rasgos descritos anteriormente detallan las formas que despliega el sistema en su implementación. Como quiera que estos enfoques tienen gran significación, hay un valor jurídico imprescindible en la estructura de los mecanismos de variabilidad en la aplicación de la justicia, como lo es el pluralismo jurídico.

Concepto que es definido por el gran jurista Norberto Bobbio, como; "El Pluralismo Jurídico basado en la Teoría de la Institución afirma que:

"El Estado no es el único centro productor de normas jurídicas sino también el producido por los grupos sociales diferentes al Estado, siempre y cuando: determinen sus fines propios, establezcan los medios para llegar a esos fines, distribuyen funciones específicas de los individuos que componen el grupo para que cada uno colabore, a través de los medios previstos, para el logro del fin y que tengan diferente cultura". (Bobbio, 2005, p 10-13).

A partir de esa idea se deja ver como el sentido de efectividad en los derechos va ligado a que existan figuras o métodos que den garantía de materialización real, y no arraigados a un solo marco dogmático. Dicho en otras palabras, el pluralismo jurídico implica que dentro de un Estado exista más de un órgano, más de una forma, más de una manera de solucionar conflictos jurídicos.

Es desde ese criterio que en Colombia encontrar esos sistemas alternativos de resolución de conflictos es una tarea de muchos años la cual se perfecciona con la inclusión positiva de cada actor participe en la gestión y administración de las herramientas de justicia; De ahí que según Rouland "El pluralismo relativiza el lugar 
del Estado y afirma la existencia de derechos no estatales, que nacieron en contextos sociales específicos. Invita a la descontextualización jurídica, espacial y temporal." (Rouland, N. 2000, p. 18)

Visto desde otra perspectiva, hay principios y fundamentos innegables no solo relacionados con la investigación y surgimiento de la norma reguladora en cuanto al sistema, sino que también exista ponderación de los derechos como principios fundantes de la justicia, que haya una reivindicación en esa construcción para que el funcionamiento del sistema esté motivado en el respeto por el cumplimiento de una garantía constitucional, o por la efectiva alternativa a un conflicto. Aduciendo este análisis, Boaventura de Sousa detalla la realidad de los derechos humanos en relación con las comunidades más aisladas y vulneradas, que son las más carentes de acceso a la justicia, o mínimos derechos fundamentales.

Al respecto Boaventura manifiesta lo siguiente:

"Hoy es innegable la hegemonía de los derechos humanos como lenguaje de la dignidad humana. Sin embargo, esta hegemonía debe convivir con una realidad alarmante. La gran mayoría de la población mundial no es sujeto de derechos humanos, sino el objeto de los discursos de derechos humanos. Por tanto, hay que comenzar por preguntarse si los derechos humanos son eficaces para la lucha de los excluidos, los explotados y los discriminados, o si, por el contrario, la hacen más difícil". (De Sousa, B. 2014. p. 23)

El papel de la responsabilidad en el cumplimiento de los derechos va de la mano con posibilitar la cesación de la vulneración a dichas afectaciones, por eso la medida básica es permitir que no se vea disminuido el rol individual y colectivo de los individuos pertenecientes a un Municipio en condición desfavorable en materia de justicia. 


\section{Discusión.}

En la discusión planteada en el desarrollo de este artículo, se busca describir lo siguiente:

A. Como los sistemas locales de justicia dan respuesta a la desarticulación institucional de las entidades territoriales. Inicialmente, el Sistema está compaginado para integrar varias formas de administración de justicia y que a través de cada competencia de los servidores públicos o particulares se puedan resolver de forma conjunta una situación particular.

Por consiguiente, el papel de resolución no será visto de manera centralizada y con la figura estatal como proveedor y proveedor de soluciones, sino que, en casos específicos, a través de la misma comunidad o contexto en particular se regularán comportamientos por medio de conciliadores en equidad, esto describiendo el rol de la Justicia Comunitaria, que en la investigación de justicia comunitaria y sociedad nacional del profesor Edgar Ardila es definida como:

"El conjunto de instancias y procedimientos mediante los cuales, para situaciones de controversia, se regulan los comportamientos legítimos a partir de normas propias de una comunidad o contexto cultural específico. Es administración de justicia desde la comunidad, a partir de sus propias reglas y principios. Es administración de justicia en tanto se desenvuelve en el ámbito de la regulación social: actúa sobre referentes normativos que la preceden y produce mandatos en los conflictos específicos. Es comunitaria en tanto su capacidad regulatoria deriva de dinámicas de identidad y pertenencia a lo mismo. Tal sentimiento puede ser ocasionado por afectos 0 tradición. (Ardila, E. 2010, p.10) 
Así pues, los sistemas locales de justicia constituyen una red de ciudadanos que pueden ser funcionarios públicos o no, que propende por conformar un equipo interdisciplinar integrado por jugadores coordinados, con vocación de apoyo y asistencia que buscan alcanzar una de las metas de esta estrategia, esta es la articulación y armonía en la resolución de conflictos jurídicos y sociales.

B. Otra de las atribuciones que se le otorga a los sistemas locales de justicia es la propuesta y plan para perseguir el adecuado y apto acceso a la administración de justicia, en cuanto que pretende la correcta preparación y potencialización de los funcionarios de la administración, toda vez que, permite que mejoren en ciertas habilidades y temáticas de conflicto; todo esto, teniendo en cuenta que se capacitará tanto a inspectores como a jueces en función del acceso a la justicia, aspecto que permitirá que los operadores de justicia apliquen de una misma forma en la vía o manera de dirimir un hecho, lo que permite un trabajo articulado y mejorado de las funciones individuales. Además de esto, se buscará multiplicar la presencia de autoridades locales, con la formación de conciliadores en equidad relacionados con la Justicia Comunitaria anteriormente detallada.

Es decir, bajo lo descrito anteriormente, se vislumbra la respuesta que los sistemas locales de justicia suministran a la problemática de incapacidad, ineficiencia, ineficacia y falta de preparación de algunos funcionarios públicos, esto con el objetivo de materializar los principios que rigen la función administrativa descritos en el artículo 209 de la Constitución Política de Colombia, y desarrollado por la ley 489 de 1998 "Por la cual se dictan normas sobre la organización y funcionamiento de las entidades del orden nacional, se expiden las disposiciones, principios y reglas generales para el ejercicio de las atribuciones previstas en los numerales 15 y 16 del artículo 189 de la 
Constitución Política y se dictan otras disposiciones". En: (Diario Oficial № 43, 46).

C. Otro de los propósitos de los sistemas locales de justicia es el fortalecimiento de la confianza en las instituciones. Para nadie es un secreto que los órganos de carácter público tienen un nivel de descrédito y desaprobación muy elevado, así lo dejó ver la encuesta realizada por la firma Gallup Colombia Ltda., entre los días 19 y 29 de agosto del 2017 en donde las cifras arrojaron que un $83 \%$ de los ciudadanos no creen en el sistema judicial. (Gallup Colombia LTDA. 2017).

Este altísimo nivel de desafección y repudio hacia la institucionalidad es la que pretende reparar los sistemas locales de justicia; y la fórmula para hacerlo es acercando lo formal, lo legal, el establecimiento en si a los ciudadanos del común como quiera que, los apropia de los medios o mecanismos de solución de conflictos, de esta manera los convierte en los protagonistas de la justicia. Estos postulados no son más que una garantía de cumplimiento del mandato constitucional contenido en el artículo 2, de la constitución del 91, el cual se refiere a los fines esenciales del Estado.

D. Otro punto que no se puede soslayar en el interior de esta discusión es, la implicación del conflicto armado en el deterioro de las instituciones y de los liderazgos sociales, toda vez que la presencia permanente de las organizaciones al margen de la ley permear y en muchos casos, reemplazaron al Estado social de derecho, es decir, estas organizaciones usurparon los tres poderes públicos en más de un municipio o ente territorial en general.

Con el advenimiento del proceso de paz y la firma del acuerdo final se crearon estrategias y métodos que dieran solución al problema de la denominada Justicia Paralela y una de esas soluciones son precisamente los sistemas locales de justicia. Con su implementación se garantiza que sea el Estado a través de las 
instituciones el que administre justicia, recuperando de esta manera los poderes y potestades que nunca debió perder y que le son inherentes.

\section{Conclusiones}

Con la finalidad de culminar el siguiente artículo, se plantean las siguientes conclusiones:

1. Los sistemas locales de justicia articulan a las autoridades jurídicas y sociales permitiéndoles colaborar conjuntamente en la formulación de soluciones a los distintos conflictos o problemas de índole legal y comunitaria, que inexorablemente se presentan en una sociedad. Su esquema cooperativo permite discutir y elegir la ruta de solución que resulte más rauda.

2. Otro de los beneficios de la implementación de los sistemas locales de justicia es la ampliación de la oferta local y mejoramiento de la funcionalidad de los actores locales, toda vez que los capacita, los acerca al derecho respetando su contexto social y cultural, además darle una visión de la realidad mucho más holística, llevándolos a comprender los conflictos desde las distintas perspectivas que ofrece el sistema.

3. Si bien es cierto, que este mecanismo obedece a realización de un proyecto desarrollado por el Ministerio de Posconflicto y PNUD como estrategia de respuesta rápida a los vacíos jurídicos y sociales que surgieron en el marco de la implementación de los acuerdos de la Habana, en donde se establecen unos Municipios priorizados que en primera medida son los beneficiarios de esta propuesta institucional, es por esta razón que en el presente artículo se propone elevarlo a categoría de política pública con lo que esto implica, esto es, incluirlo 
dentro de los planes de desarrollo de los distintos entes territoriales y que haga parte del ejercicio normal de las funciones de los operadores judiciales con el fin de que se ejecute en todo el territorio nacional y se contribuya a la superación de los problemas de acceso a la justicia y confianza institucional.

\section{Referencias.}

Ardila, Édgar (2017). Caja de Herramientas de los Sistemas Locales de Justicia segunda Edición. Bogotá: Chigüiro estudio.

Buelvas Mendoza, M., \& Florez Campo, O. (2017). Elementos para la discusión sobre la gobernanza y gobernabilidad en el posconflicto en Colombia. Erg@omnes, 9(1), 145-168. https://doi.org/10.22519/22157379.1016

Centro de justicia de las Américas. Justicia Comunitaria y Sociedad Nacional (En línea)

https://www.cejamericas.org/Documentos/DocumentosIDRC/135justiciacom unitariaysociedadnacional.pdf. (Citado el 26 noviembre de 2017)

Colombia. Constitución Política de 1991.

Colombia. Congreso de la República. Ley 489 del 29 de diciembre de 1998 "Por la cual se dictan normas sobre la organización y funcionamiento de las entidades del orden nacional, se expiden las disposiciones, principios y reglas generales para el ejercicio de las atribuciones previstas en los numerales 15 y 16 del artículo 189 de la Constitución Política y se dictan otras disposiciones". En: Diario Oficial № 43, 46.

Colombia. Consejo Superior de la Judicatura. Acuerdo № PSAA12-9139 del 17 de enero del 2012 "Por el cual se adopta el Plan Especial de Descongestión de la Jurisdicción de lo Contencioso Administrativo".

Corporación excelencia en la justicia. La CEJ revela alarmantes cifras de congestión judicial.

(En línea).

Revista Legem, Universidad del Atlántico 5 (2), Julio - Diciembre 2019, pp. 23-41 
http://www.cej.org.co/index.php/sala/noticiascej/788-la-cej-revelaalarmantes-cifras-de-conjestion-judicial (Citado el 04 de diciembre de 2017).

De sousa, Boaventura. (2014). Derechos humanos, democracia y desarrollo. Bogotá: Centro de Estudios de Derecho, Justicia y Sociedad, Dejusticia.

García Villega, Mauricio s, Espinosa Restrepo, José Rafael, Lalinde Ordóñe, Sebastiánz, Arroyave Velásquez y Lina María, Villadiego Burbano, Carolina. (2015). Casas de Justicia: una buena idea mal administrada. Casas de Justicia. Bogotá: Centro de Estudios de Derecho, Justicia y Sociedad, Dejusticia.

Programa de naciones unidas para el desarrollo. Proyecto: Asistencia técnica para la creación y fortalecimiento de los Sistemas Locales de Justicia en 13 municipios priorizados en Colombia. (En línea) http://mptf.undp.org/document/download/16887. (Citado el 10 de noviembre de 2017)

Ministerio de justicia. Cartilla SLJ (En línea) https://www.minjusticia.gov.co/Portals/0/Sistemas\%20Locales\%20de\%20Ju sticia/Doc/CartillaSLJ.pdf. \{Citado el 20 de noviembre de 2017\}

Bobbio, Norberto (2005). Teoría general del derecho. Bogotá: Temis.

Rouland, Norbert. (2000). L'antropologie juridique, París, PUF.

Gallup Colombia LTDA. Encuesta (En línea) http://media.caracoltv.co/Noticias/GALLUP\%20POLL\%20FAVORABLEDESFAVORABLE.pdf. (Citado el 04 de diciembre de 2017). 